\title{
RENAL MYXOMA: AN UNEXPECTED DIFFERENTIAL DIAGNOSIS
}

\author{
Carlos Gómez-González ${ }^{1}$, Virginia Moreno-Nieto ${ }^{1}$, Mikel Vicente-Pablos ${ }^{2}$, \\ IVÁn FernandeZ-VeGa ${ }^{1}$
}

\author{
${ }^{1}$ Service of Anatomic Pathology, University Hospital of Araba-Txagorritxu, Vitoria-Gasteiz, Spain \\ ${ }^{2}$ Service of Radiology and Magnetic Resonance, OSATEK. S.A., Unidad de Vitoria-Gasteiz, \\ University Hospital of Araba-Txagorritxu, Spain
}

\begin{abstract}
Myxomas are rare mesenchymal tumors that can appear in many anatomical locations, although they are mainly seen in heart and skin. To date, only twelve cases of pure renal myxomas have been reported in the literature. We describe a case of a young Cuban woman with an asymptomatic irregular cyst lesion in her left kidney which was eventually diagnosed as renal mixoma. We also provide radiological and pathological studies.
\end{abstract}

Key words: kidney, magnetic resonance imaging, myxoma.

Myxomas are rare mesenchymal tumors that can appear in many anatomical locations, although they are mainly seen in heart and skin [1]. They can also be part of the Carney complex (myxomas, spotty pigmentation, endocrine overactivity, and schwannomas) [2]. To date, twelve cases of pure renal myxomas have been reported in the literature [3]. We describe a clinicopathological study of a young Cuban woman with mesangial proliferative glomerulonephritis and renal insufficiency who had an asymptomatic Bosniak IV cystic lesion in her left kidney.

We present a 29-year-old woman diagnosed with mesangial proliferative glomerulonephritis in 2005 . Then, she developed renal insufficiency and she was treated with inbibitors of the renin-angiotensin-aldosterone system (RAAS). In 2011, a routine checkup including magnetic resonance imaging (MRI) was performed and an asymptomatic small cystic lesion in the interpolar region of her left kidney was found. After a year of follow-up, a new MRI was done in which the lesion appeared increased. Meanwhile, the patient became pregnant. Then, between the $2^{\text {nd }}$ and $3^{\text {rd }}$ trimester, she developed diastolic hypertension treated with methyldopa. No problems during delivery were reported. A last routine MRI was performed the present year, where the cystic lesion was eventu- ally classified as Bosniak IV, showing a maximum diameter of $4.4 \mathrm{~cm}$ and located close to the renal hilum (Fig. 1, white arrow). Then, she underwent a robotic partial tumorectomy through transperitoneal access, causing a significant abdominal wall hematoma and anemia, completely resolved using four bags of packed red blood cells.

The resected surgical specimen weighed $45 \mathrm{~g}$. On sagittal section, a $4.5 \mathrm{~cm} \times 4 \mathrm{~cm}$ pseudoencapsulated tumor with a narrow area of adjacent unaffected renal parenchyma was seen. The mass showed gray-white semi-translucent and gelatinous areas admixed with some brownish zones (Fig. 2A). Histologic examination revealed a tumor constituted by fibroblast-like, spindle cells with myxoid stroma. Some hypercellular areas were noted. Nonetheless, the majority of the tumor was paucicellular and hypovascular, intermingled with a large amount of basophilic interstitial mucoid material and occasional fine strands of fibrous tissue. No evidence of atypical mitotic figures or necrotic areas was found (Fig. 2B). On immunohistochemistry, the spindle cells stained strongly positive for vimentin and alpha-smooth muscle actin (Fig. 2C and 2D respectively); weakly positive for CD99 (Fig. 2E); and negatively for multi-cytokeratin (AE1/ AE3), EMA, S100, desmin, CD34, CD31, p53 and 

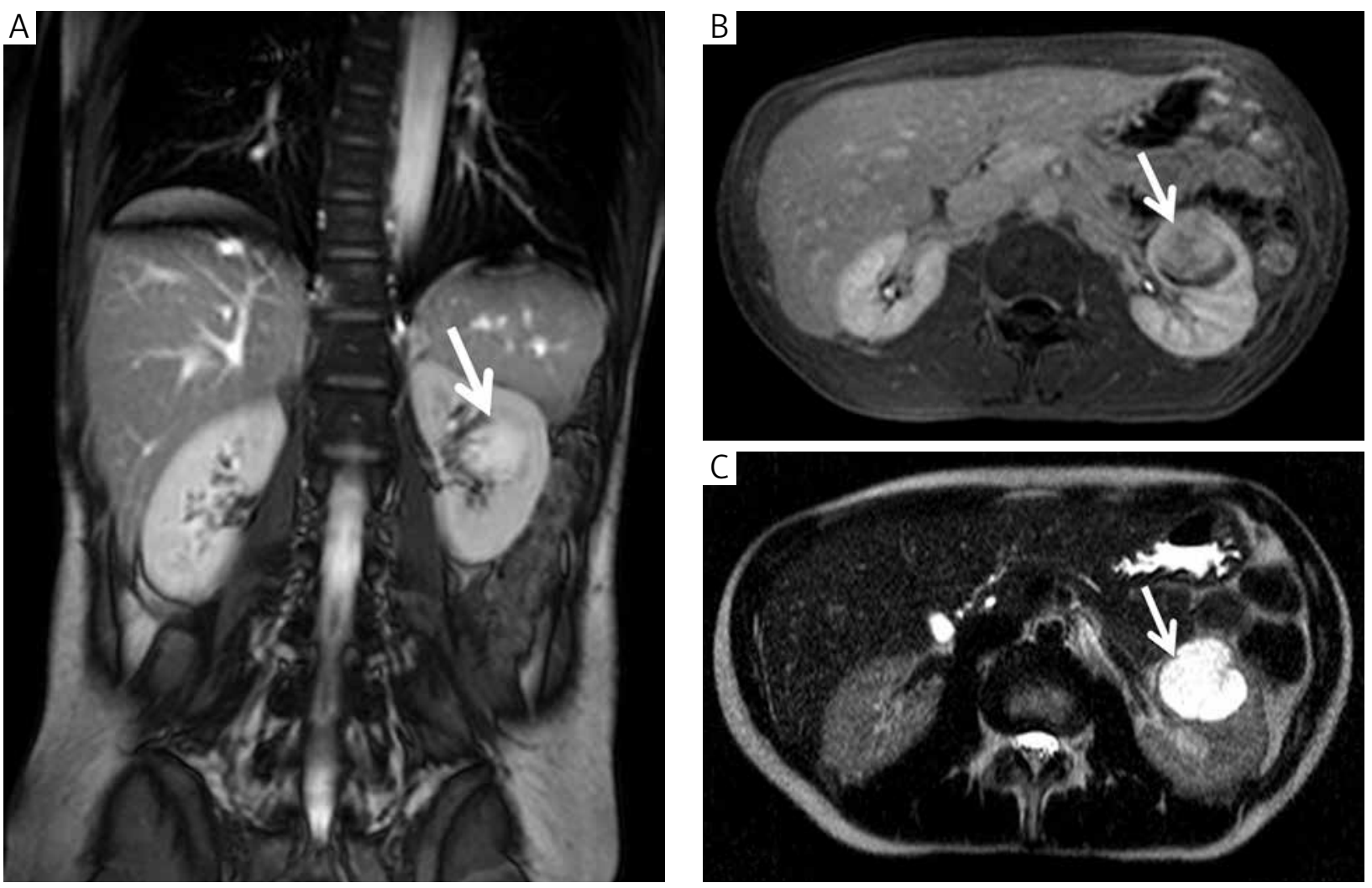

Fig. 1. Magnetic resonance images pointing out the cystic lesion within the left kidney (white arrow). A, B) Contrast-enhanced T1-weighted image in venous phase shows a low intense renal mass (coronal and transverse view respectively). C) T2-weighted image shows a hyperintense renal mass (transverse view)

Bcl2. The Ki-67 index was very low (less than 1\%). Taking into account these histological and immunohistochemical appearances, the final histopathological diagnosis was primary intrarenal myxoma.

Although an accurate histopathological diagnosis of this benign tumor is not very difficult using basic morphologic criteria, a full differential diagnosis has to be made considering other possible renal benign mesenchymal tumors such as leiomyoma, hemangioma, lymphangioma, neurofibroma, solitary fibrous tumor, schwannoma and glomus tumor. Furthermore, renal myxomas should also be differentiated from other benign and malignant mesenchymal tumors with myxoid transformation, considering myxoid neurofibroma, myxoid leiomyoma, myxolipoma and myxoid variant of malignant fibrous histiocytoma [3]. Our case did not show different histological areas such as neural or leiomyomatous changes.

Radiologically, the tumoral lesion was classified as Bosniak IV by the radiologist according to the Bosniak Classification of Renal Cystic Disease, because of its irregular wall thickening and the evidence of some independent enhancing soft-tissue components [4].

In conclusion, combination and integration of clinical behavior with radiology are usually in agreement in malignant tumors. However, this case highlights the major relevance of histological examination in order to eventually classify every tumor as benign or malignant, especially when treatment differs significantly.

The authors declare no conflict of interests.

\section{References}

1. Durgut K, Onoglu R, Gormus N. Primary cardiac myxomas: report of 28 cases and review the literature. J BUON 2011; 16: $345-8$.

2. Courcoutsakis NA, Tatsi C, Patronas NJ, et al. The complex of myxomas, spotty skin pigmentation and endocrine overactivity (Carney complex): imaging findings with clinical and pathological correlation. Insights Imaging 2013; 4: 119-33.

3. Yildirim U, Erdem H, Kayikci A, et al. Myxoma of the renal sinus: case report and literature review. Turk Patoloji Derg 2012; 28: 76-9.

4. Whelan TF. Guidelines on the management of renal cyst disease. Can Urol Assoc J 2010; 4: 98-9.

\section{Address for correspondence}

Dr Iván Fernández-Vega MD, $\mathrm{PhD}$

Service of Anatomic Pathology

Hospital Universitario de Araba-Txagorritxu,

C/Jose Achotegui s/n, E-01009, Vitoria-Gasteiz, Alava, Spain tel. 34945005107

e-mail: ivan_fernandez_vega@hotmail.com 

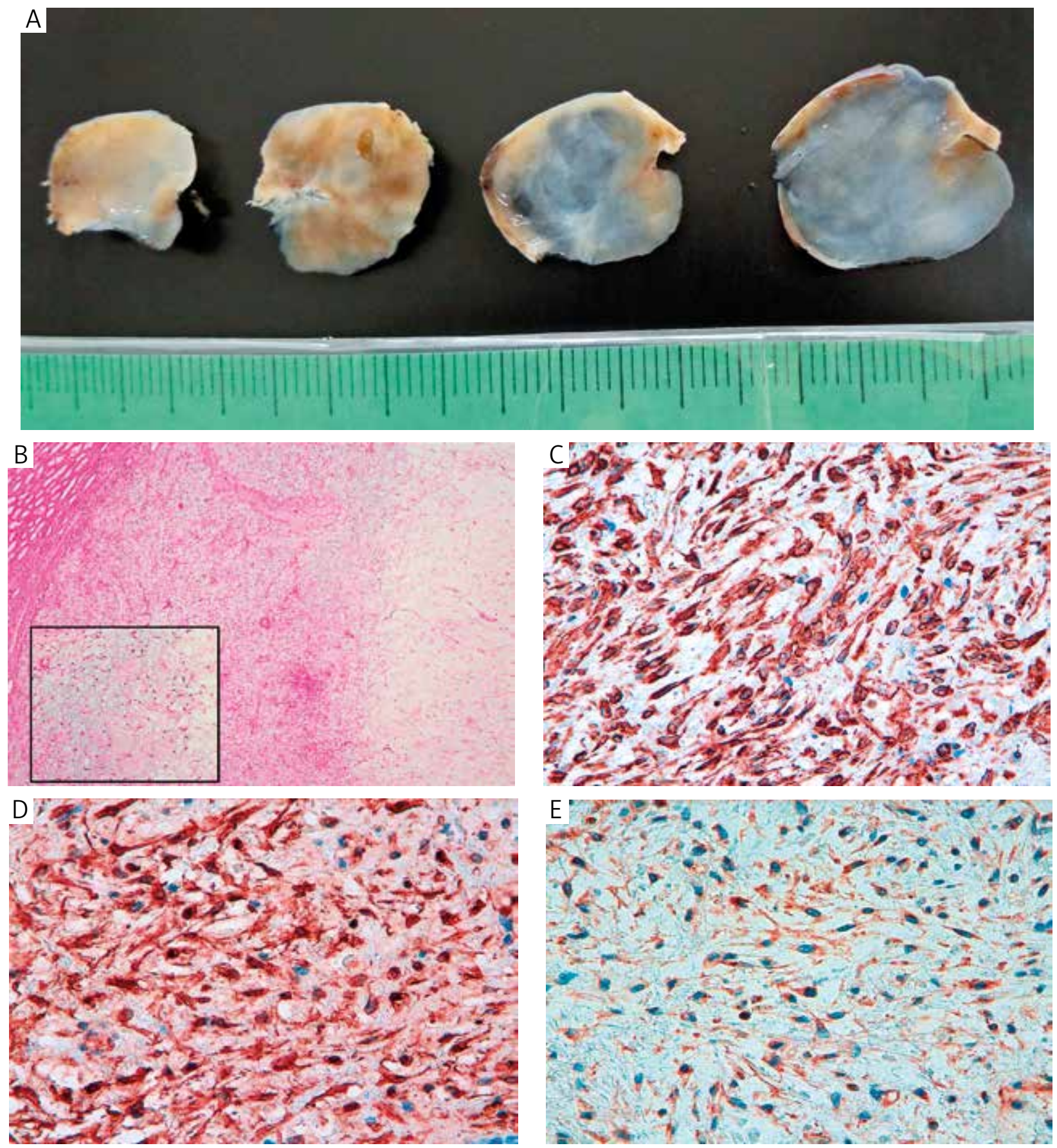

Fig. 2. A) Sliced gross pathologic examination shows a well-circumscribed renal tumor with a soft to solid, semi-translucent gelatinous myxoid appearance with gray-white areas admixed with some brownish zones. B) Microscopic findings of the tumor. Low-power view showing a pseudoencapsulated myxomatous tissue constituted by fibroblast-like spindle cells. Some hypercellular areas are noted in peripheral zones. Large amounts of basophilic interstitial mucoid material are seen. Remaining renal parenchyma is noted on the left upper corner. The inset shows better details of the myxoid mass. (HE, magnification 20×). C) Immunohistochemistry for vimentin presents strong cytoplasmic positivity in tumor cells (magnification $400 \times$ ). D) The tumor cells display strong staining for alpha-smooth muscle actin (magnification $400 \times$ ). E) The fibroblast-like cells of the tumor are weakly positive for CD99 (magnification $400 \times$ ). All immunostaining was performed by immunoperoxidase technique on paraffin-embedded tissue sections 